\title{
Time displacement for a wall-stabilized electric arc in a transverse magnetic field.
}

\author{
J. R. Ramos-Barrado and P. Galan-Montenegro \\ Departamento de Física Aplicada. Facultad de Ciencias. Universidad de Málaga, E-29071 Málaga, Spain
}

(Reçu le 9 décembre 1986, révisé le 18 février 1987, accepté le 16 avril 1987)

\begin{abstract}
Résumé. - Le temps mis par un arc électrique pour arriver à sa position de déviation sous l'influence d'un champ magnétique transversal est mesuré pour des arcs de différentes puissances. Le temps mis par l'arc varie linéairement avec la déviation quand on prend comme paramètre le quotient entre le champ magnétique appliqué et le champ électrique dans la colonne de l'arc.
\end{abstract}

\begin{abstract}
The time taken for a wall-stabilized electric arc in a transvèrse magnetic field to reach its displacement position are measured for different arc powers and pressures. This time is linear with the displacement position, and its coefficient is the ratio between the applied magnetic field and the electric field in the arc column.
\end{abstract}

\section{Introduction.}

Many investigators have studied the behaviour of electric arc in a transverse magnetic field. The published works deal with a wide variety of phenomena which may be grouped under three heading : thermal, electrical and mechanical (Sauter [1], Rosenbauer [2], Simon [3]).

In studies of a wall-stabilized electric arc, the usual subject are the magnetohydrodynamic phenomena associated with the Lorentz force, which cause deviation of the column from its axial position (Seeger [4, 5], Raeder et al. [6], Sauter [1]) and its subsequent return in a finite time, when the transverse magnetic field is removed (Ramos-Barrado [7]).

The simplest method of producing displacement of an electric arc is to apply a transverse magnetic field; this is frequently employed in arc welding techniques and in DC arc circuit-breakers (Novak et al. [8], Sen and Das [9], Yasko [10], Nagamatsu and Symolon [11], Nagamatsu and Whang [12], Meier et al. [13]). Although these arcs are usually cooled by surrounding them with an axial argon gas jet; the thermal dynamic conditions are very close to those obtaining in electric arcs in water-cooled chambers.

The aim of the present paper is to study the movement of the column of a long electric arc from its axial position, maintained under the influences of continuous, external and stationary, transverse magnetic field; and to determine the relationships between the displacement times and the different characteristic factors of the arc.

Section 2 describes the experimental method employed to measure arc displacement and the time taken for the arc to reach the displaced position after applying the external transverse magnetic field.

Section 3 contains the experimental results and conclusions.

\section{Experimental method.}

The discharge chamber was a water-cooled quartz tube $10 \mathrm{~mm}$ radius : we used cylindrical wolfram electrodes and the gas was argon of $99.996 \%$ purity. The arc was switched on by connecting the electrodes. They were displaced by a rack system (Fig. 1). All experiments were made without axial gas flow.

The $E(I)$ characteristic was determined by measuring the voltage between the end of each electrode for arcs of different lengths, and by establishing a linear fit whose slope is the strength of the electric field. The confidence level of the fit for every straight line is greater than $99 \%$. Figure 2 shows the $V(I)$ arc characteristic for the pressure of $1 \mathrm{~atm}$ and a gap between electrodes of $29.7 \mathrm{~cm}$. Figure 3 shows the $E(I)$ characteristic for the pressure values : $0.5,1$ and 2 atm. 


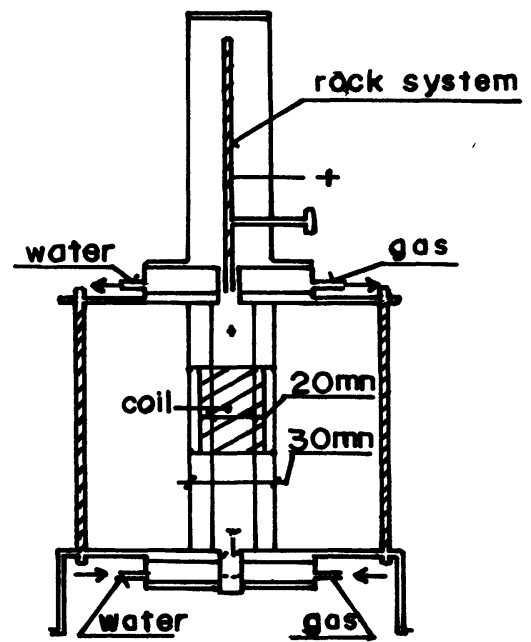

Fig. 1. - Scheme of the electric arc.

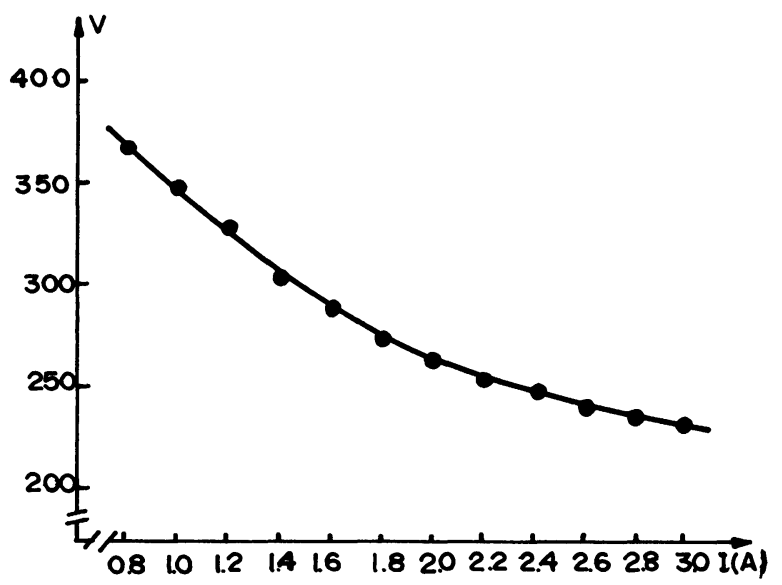

Fig. 2. $-V(I)$ arc characteristic (gap between electrodes $29.7 \mathrm{~cm})$.

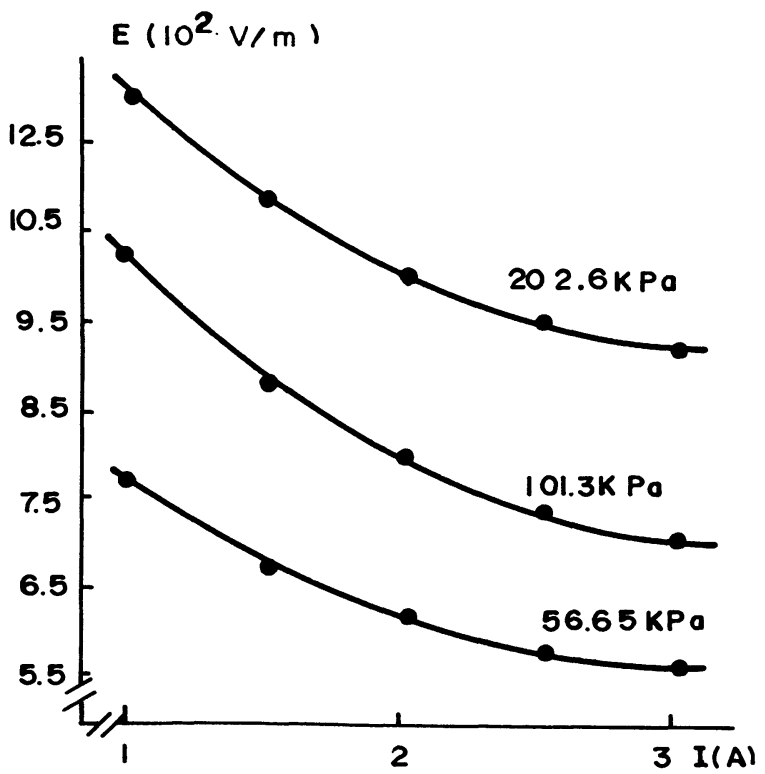

Fig. 3. $-E(I)$ arc characteristic.
The transverse magnetic field was produced by two equal coils of $10 \times 4.5 \mathrm{~cm}$ located in line and opposite one another on the outer tube, their inner edges were located $10 \mathrm{~cm}$ of the end of each electrodes ; so that, only the central section of the arc column was under the effect of the transverse magnetic field and the electrode effect did not perturbe the arc column displacement. We measured the magnetic field strength with a Gaussmeter giving an accuracy of $1 \%$. In the central section, between the two coils, the magnetic field was homogeneous over $2 \%$. The ohmic resistance $(8.1 \Omega)$ and selfinductance $\left(4.41 \times 10^{-3} \mathrm{H}\right)$ of the two coils was measured by a Universal Bridge HP2465A. We added a $200 \Omega$ resistor to the coil circuit. The time constant of this circuit was about $20 \times 10^{-6} \mathrm{~s}$. Figure 4 shows the electric current intensity in the coil circuit versus time.

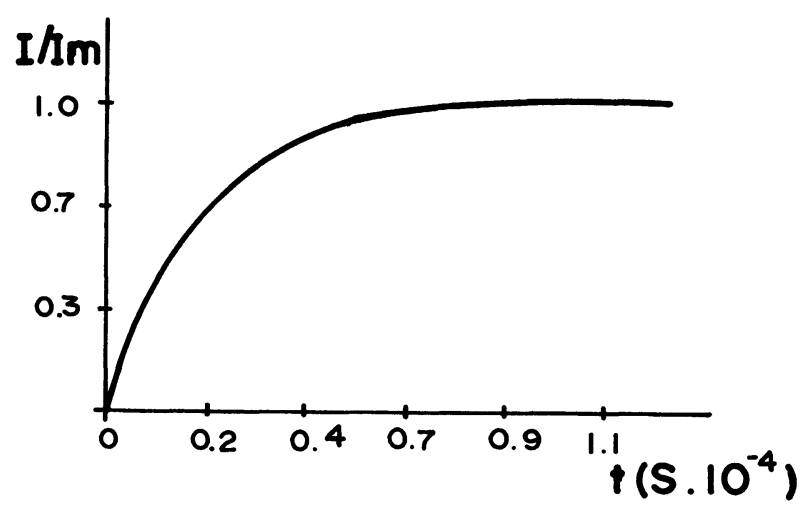

Fig. 4. - Electric current intensity in the coil circuit vs. time.

Arc displacement was measured by photographing the magnetically deflected arc and the arc in the axial position in the same photogram on a Kodak film of 9 DIN sensibility. The magnification was $1 / 2$. Displacement measurements were carried out on the photogram using a Zeiss IV Stereomicroscope with an accuracy of $0.1 \mathrm{~mm}$ (Ramos-Barrädo [14]).

Time measurements taken for the arc to reach its displacement position began from the time of switching on the magnetic field to the time that the arc stimulate a photoelectric cell placed with an accuracy of $0.2 \mathrm{~mm}$ in the arc image position of an optical device designed to eliminate the halo of the arc column. This optical system consisted of an $85 \mathrm{~mm}$ focal length lens placed in the head of a variable length black box; a diaphragm was placed at the focal length to eliminate a non-parallel ray and to give the clearest possible image and the LDR located on the image plane in the black box. The optical device is centred on the undisplaced arc axis.

The signal from the photoelectric resistance cell is processed by an electronic circuit consisting on a 


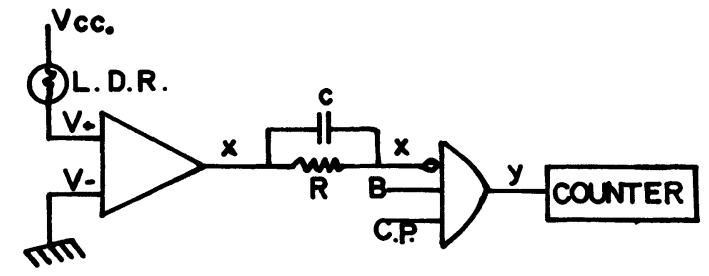

Fig. 5. - Arc time deviation measurement circuit (C.P. : Clok Pulses ; B : coil circuit signal ; LDR : photoelectric cell).

differential voltage comparator and $a$ « $Y$ » gate (Fig. 5). The ohmic resistance of the LDR in darkness was $200 \mathrm{M} \Omega$ and when it was exposed to the arc ligth was only $20 \mathrm{M} \Omega$; and this resistance was independent of the electric arc current. The LDR signal is applied to the differential comparator, whose output is inverted and then applied to one of the inputs of the " $Y$ » gate. Clock pulses from a HP3310A function generator, with a frequency of $10^{5} \mathrm{~Hz}$, and the signal from the coil circuit were also applied to the « $\mathrm{Y}$ » gate. The gate only permits the clock pulses to pass when there is magnetic field, without a LDR cell signal. These signals are blocked when the LDR is stimulated. The clock pulses are counted by a digital counter which gives a $50 \mathrm{~ns}$ resolution.

This method requires constant response times for the coil and LDR-comparator system and these times must be lesser than the measurement time.

The intensity delay time at coil circuit is very small (Fig. 4) ; the intensity reaches $99 \%$ in $90 \times 10^{-6} \mathrm{~s}$; 500 times less than the smallest measurement time (Fig. 7).

The time measurements are made at the zero level at the differential comparator and LDR,-and are monitored by an oscillograph HP182C.

\section{Results and conclusions.}

The arc displacement and the time taken to reach the displaced position was measured for current intensities of $1,1.5,2,2.5$ and $3 \mathrm{~A}$; gas pressure of 50.6, 101.3 and $202.6 \mathrm{kPa}$, and magnetic fields between $0.25 \times 10^{-4}$ and $10^{-3} \mathrm{~T}$. Figure 7 shows the arc deviation an as function of both the applied magnetic field and the applied electric current.

The arc displacement is produced by the Lorentz force originated by the applied transverse magnetic field ; but a Lorentz force also produces arc displacement as a function of the arc power. For a given Lorentz force, the displacement time did not increase with the arc displacement. This behaviour is caused by the double effect, in that the transverse magnetic field to originates a mass flow in the same sense as the Lorentz force ; but this mass flow at the same time implies a distortion of the symmetrical temperature distribution of the undisplaced electric arc and

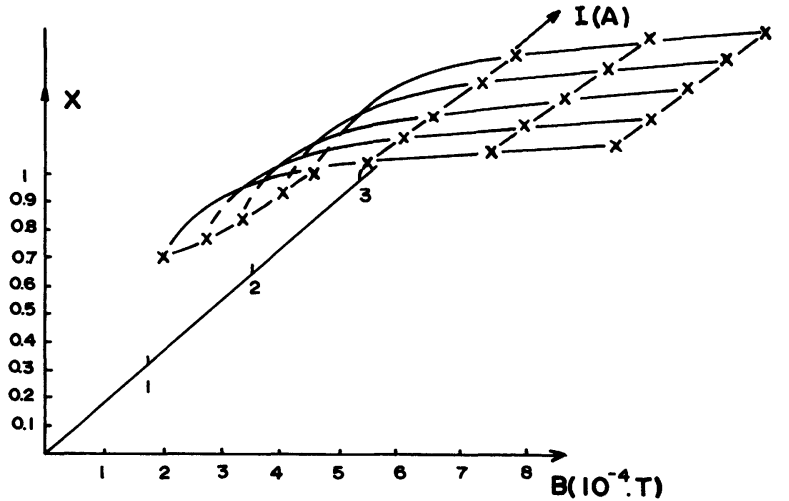

Fig. 6. - Relative arc displacement vs. magnetic field and electric arc current $\left(X=X_{\mathrm{A}} / R\right)$.

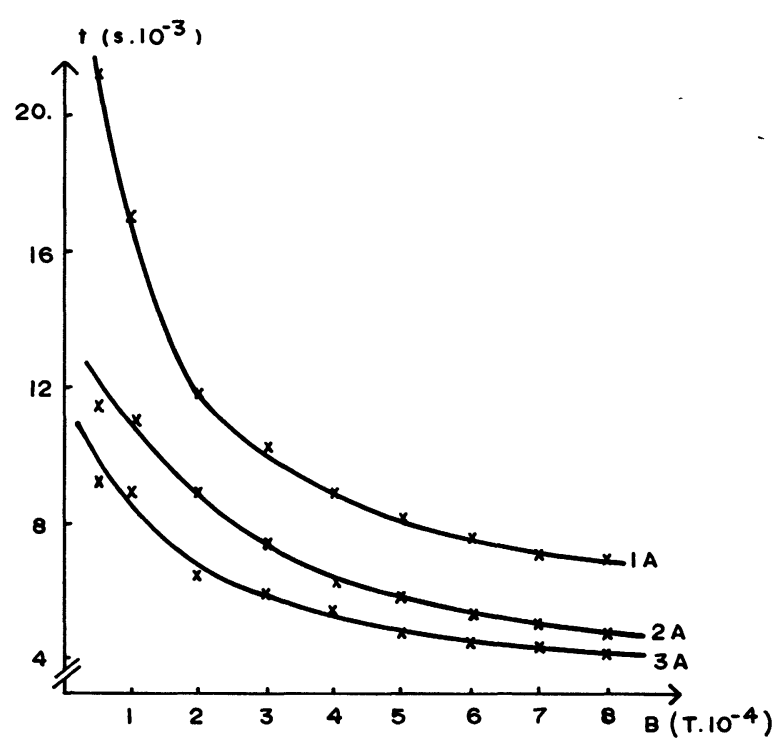

Fig. 7. - Time displacement vs. magnetic field.

the creation of the grad $\left(\nabla^{2} S\right), S$ is the transfer heat function (Schmitz [15]), which opposes to the displacement of the maximum temperature point from its axial position and gives rise to an interaction between the mass flow and a «thermodynamic force " which delays arc displacement (Maecker [16]) relative to the mass flow generated by the Lorentz force.

The time taken to reach the deviated position increases rapidly as the transverse magnetic field is diminished because of the decrease in the mass flow velocity (Fig. 6) originated by the Lorentz force (Ramos-Barrado [17]).

Furthermore, the decrease in arc displacement time resulting from an increase in current intensity is caused by the fact that the mass flow velocity is increase more rapid than the increase in value the resistance to deformation of the isothermal field. These conclusions are confirmed by the measurements of the average velocity of the arc displacement (Fig. 8). 


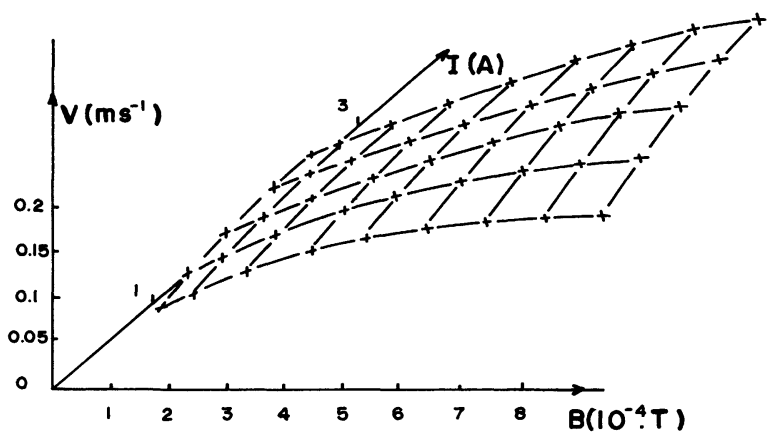

Fig. 8. - Mean velocity of the arc displacement $v s$. magnetic field and electric current intensity.

In agreement with the above argument, the mass flow velocity increases with the arc power for a given pressure and applied magnetic field (Sauter [1]) ; similarly, for a given pressure and arc displacement, the increase of the arc power increases the grad $S$. Consequently, the applied Lorentz force may by normalized by dividing it by the arc power $(I E)$, calculated from the $E(I)$ characteristic. Figure 9 shows the time displacement $v s$. relative arc displacement for several values of the normalized Lorentz force $(B / E)$.

In this way, for a given Lorentz force and a given arc power, the arc displacement time increases with the arc deviation and also, for a given displacement, the arc displacement time increases inversely with the ratio between the Lorentz force and the arc power.

The electric field in the arc column increases with the applied magnetic field (Sauter [1], Ramos-Barrado [18]), (Fig. 10 shows the electric field plotted

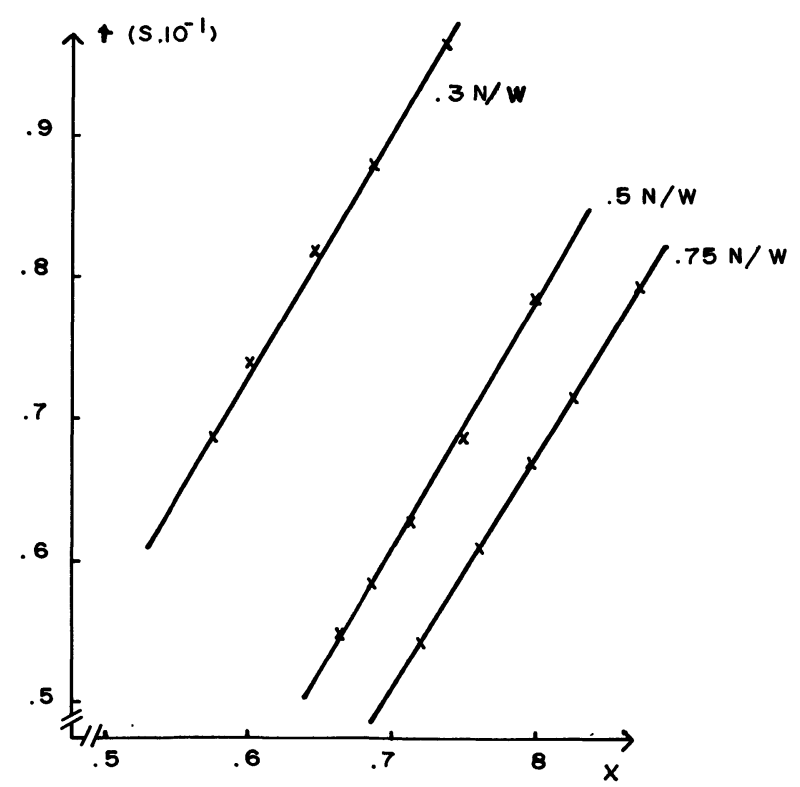

Fig. 9. - Time displacement vs. relative arc displacement for different ratios $B / E$.

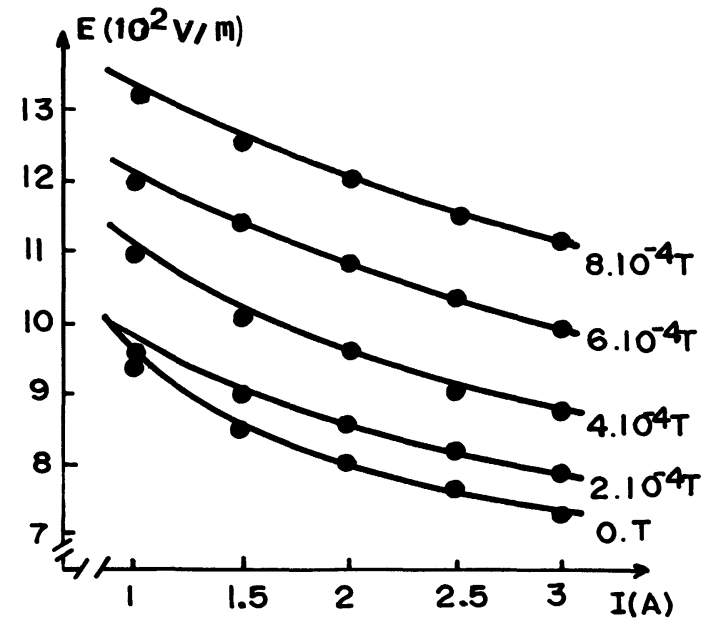

Fig. 10. - Electric arc increase vs. applied transverse magnetic field.

against the external magnetic field for some arc current intensities). However, this increase of the arc power does not increase the axial temperature in a magnetically deflected arc (Sauter [1]) ; because the excess of arc power is dissipated by the viscous mass flow, and the resistance to deformation of the isothermal field does not increase. So that the electric field of the undisplaced arc appears to be more representative of the thermal resistance.

The results were fitted for each value of the coefficient $B / E$ by a linear regression (Lybanon [19]), as follows :

$$
t_{\mathrm{d}}(x, B / E=\text { Const. })=a x .
$$

The degree of significance is greater than $99 \%$. The parameter « $a$ » varies with the ratio $(B / E)^{-1}$ and was fitted by the same method [16] to another linear equation which also gives degree of $99 \%$ significance.

In this way, the arc displacement time can be expressed as a function of both the arc relative displacement and the ratio between the external applied magnetic field intensity and the electric field intensity, in the arc column :

$$
t_{\mathrm{d}}(x, B / E)=A(B / E)^{-1} x .
$$

The experimental results were fitted to (2) by the Fletcher-Powell method and gave a $99 \%$ confidence level for

$$
A=(379 \pm 10) 10^{-4} \mathrm{~s}^{2} / \mathrm{m} .
$$

If we consider both a given electric field and a given displacement, the arc displacement time diminishes as the magnetic field increases. And, if we consider a given displacement and a given magnetic field, the arc displacement time increases with the increase of the electric field.

The increase of pressure produces the following 
effects on the arc displacement time : a) the mass flow velocity and the displacement time decrease slightly at the maximum temperature [14]; and b) the arc power increase $(I E)$, together with an increase in the time taken for the arc to reach its maximum displacement.

\section{References}

[1] SAuter, K., Z. Naturforsch. 24A (1969) 571.

[2] Rosenbauer, H., Z. Phys. 245 (1971) 295.

[3] Simon, M., Freir Bogen in Quermagnetfeld, Thesis, Technical University, Munich (G.F.R.) (1967).

[4] Seeger, G., Z. Angew. Phys. 25 (1968) 23.

[5] Seeger, G., Z. Angew. Phys. 29 (1970) 375.

[6] Raeder, J., Seeger, G., Gorenflo, H., Phys. Fluids 17 (1974) 137.

[7] Ramos-Barrado, J. R., Contr. Plasma Phys. 26 (1986) 313.

[8] NovaK, J. P., Fuchs, V., Proc. IEEE 121 (1974) 81.

[9] Sen, S. N., DaS, R. P., Int. J. Electron. 34 (1973) 527.

[10] Yasko, O. I., Int. J. Heat Mass Transfer. 14 (1971) 1983.

[11] Nagamatsu, H. T. and Symolon, P. D., $A I A A$ (81) 0097, AIAA 19th Aerospace Sciences Meeting, St. Louis (1981).
[12] Nagamatsu, H. T. and Whang, H. C., $A I A A$ (86) 1090, AIAA 4th Fluid Mechanics, Plasma Dynamics and Laser Conference. Atlanta (1986).

[13] Meier, R., Kneubuhl, F. K., Coccioni, R., Weys, H., Fischer, E. and Schotzau, H. J., IEEE Trans. Plasma Sci. PS-14 (1986) 390.

[14] Ramos-Barrado, J. R., Monterde-Garcia, A., Pardo-SAnchez, G., An. Fis. 79B (1983) 81.

[15] Schmitz, G., Z. Naturforsch. 5A (1950) 571.

[16] Maecker, H., Proc. IEEE 59 (1971) 439.

[17] Ramos-Barrado, J. R., Acta Phys. Hung., to appear.

[18] Ramos-Barrado, J. R., An. Fis. 81A (1985) 180.

[19] Lybanon, M., Am. J. Phys. 52 (1984) 22. 